\title{
Guanabenz Acetate
}

National Cancer Institute

\section{Source}

National Cancer Institute. Guanabenz Acetate. NCI Thesaurus. Code C65826.

The orally bioavailable, acetate salt form of guanabenz, a centrally-acting alpha-2 adrenergic receptor agonist, with anti-hypertensive and potential antineoplastic, cytoprotective and bone resorption inhibitory activities. Upon oral administration, guanabenz suppresses endoplasmic reticulum (ER) stress by inhibiting the stress-induced dephosphorylation of eukaryotic translation initiation factor 2 alpha (elF2a), thereby enhancing the phosphorylation level of elF2a. This causes elF2a-mediated downregulation of the Rac1 pathway, upregulates the expression of activating transcription factor 4 (AT F4), which plays a key role in osteoblastogenesis, and downregulates the expression of nuclear factor of activated T-cells, cytoplasmic 1 (NFAT C1), which is a transcription factor that plays a key role in osteoclastogenesis. This enhances osteoblastogenesis and suppresses osteoclastogenesis. Altogether, this promotes new bone formation and prevents bone degradation. In addition, guanabenz blocks the proliferation, survival, motility and invasiveness of tumor cells through the elF2a-mediated downregulation of Rac1 signaling. Rac1, a Ras-related small GT Pase belonging to the Rho family, plays a key role in tumor cell proliferation, survival and motility. 